\title{
Sampling Serum in Patients With Vitiligo to Measure Disease Activity in the Skin
}

Mehdi Rashighi, MD; John E. Harris, MD, PhD

In this issue of JAMA Dermatology, Speeckaert and colleagues ${ }^{1}$ report that levels of the soluble CD25 and CD27 molecules (sCD25 and sCD27) are elevated in the serum of patients with active vitiligo compared with patients with stable disease. In addition, sCD25 levels were $\leftarrow$ found to be significantly Related article page 1194 lower in the serum of patients being treated with topical immunosuppressants (including steroids and calcineurin inhibitors) and the serum level of sCD27 was significantly lower in patients with recent repigmentation, suggesting a potential to use these as biomarkers to monitor treatment responses. Interestingly, the authors continued to prospectively study a relatively large number of participants and demonstrated that serum levels of both sCD25 and sCD27 were associated with disease progression during follow-up. These important findings suggest that monitoring of these markers in the serum of patients with vitiligo may provide a glimpse into what is happening in the skin, a process that is not obvious by simple observation in vitiligo, in contrast to more inflammatory diseases.

CD25 is the a chain subunit of the heterotrimeric interleukin 2 (IL-2) receptor, which is expressed on the surface of activated T cells. Interleukin 2 is a cytokine with pleiotropic immune functions, largely known for its role in both inflammation and induction of immune tolerance, ${ }^{2}$ as it is a growth factor for effector $\mathrm{T}$ cells that promote inflammation, as well as for regulatory $\mathrm{T}$ cells that suppress inflammation. Soluble CD25 results from the proteolytic cleavage of the IL-2 receptor on activated $\mathrm{T}$ cells and is reportedly elevated in the serum and plasma of patients with several lymphoproliferative and autoimmune diseases. ${ }^{3}$ A recent study suggested that sCD25 might act as a decoy receptor to sequester local IL-2 in $\mathrm{T}$ cell-infiltrated peripheral tissue, and therefore diminish IL-2-mediated immune suppression, although its functional role in immune responses is still largely unclear. ${ }^{3}$ Regardless, the sCD25 level is remarkably stable in the serum of healthy individuals, supporting its use as a clinical biomarker for disease activity in inflammatory diseases. ${ }^{4}$

CD27 is a type I transmembrane protein and member of the tumor necrosis factor receptor superfamily that is expressed on the surface of specific subsets of T cells (among other immune cells), and is known for its role as a co-stimulatory receptor that promotes T-cell survival, proliferation, and generation of memory. ${ }^{5}$ Specifically, studies in both human and mouse models indicate that CD27 signaling can promote interferon- $\gamma^{-}$ dependent immune responses, which is particularly relevant for vitiligo. ${ }^{5,6}$ Soluble CD27 can be released into the blood circulation by differential splicing and shedding of the extracellular domain of the membrane-bound receptor on activated $\mathrm{T}$ cells. Interestingly, the serum sCD27 level has been used as a biomarker to monitor immune activation and disease burden in various inflammatory disorders; however, it is currently not clear whether SCD27 has a functional role in these conditions or is simply a by-product of T-cell activation. ${ }^{7,8}$

Vitiligo is a common T-cell-mediated autoimmune disease presenting clinically as symmetrical macular depigmentation caused by epidermal melanocyte loss. ${ }^{9}$ Recent studies in vitiligo pathogenesis suggest that targeting functional immune pathways may be an excellent strategy to develop new treatments. ${ }^{6,10}$ Randomized clinical trials represent the most stringent test of efficacy for investigational new drugs. However, these trials have certain limitations, particularly in regard to the large number of participants needed, the prolonged follow-up required to assess the primary study end point, and the associated costs. ${ }^{11}$ For vitiligo, time for follow-up is typically 3 to 6 months, which can make trials timeconsuming and expensive.

Adaptive trials have recently garnered attention as an alternative study design because they provide an opportunity to prospectively plan modifying 1 or more aspects of the study design, depending on an interim analysis of the results. ${ }^{12}$ This may be an optimal early study design, particularly in proofof-concept trials, in which many uncertainties about dose response, end points, and effect sizes exist. ${ }^{13}$ Importantly, biomarker or surrogate end points are considered a critical component of adaptive trials because they provide early indication of drug efficacy and enable interim analysis for adaptation of the trial as needed. ${ }^{11}$ It is therefore crucial to identify and validate biomarkers that can be used in future trials to test new investigational drugs in vitiligo.

Another recent study reported that serum levels of the interferon- $\gamma$-dependent chemokines CXCL9 and CXCL10 reflected disease activity and treatment response in vitiligo, ${ }^{14}$ and the present study now adds additional markers for consideration to be used in future clinical trials and other clinical studies. Advantages of this particular study include the large number of participants enrolled, allowing subset analyses of disease activity and treatment response, as well as its prospective design. The authors ${ }^{1}$ did not find any correlation between serum levels of these soluble molecules and the extent of disease as defined by the vitiligo body surface area affected. This is in contrast to a previously published study of SCD25 in a smaller number of participants. ${ }^{15}$ 
Like many other reports of biomarkers in vitiligo, ${ }^{6,14} 1$ limitation of this study is that it relies on serum markers rather than using the tissue directly. Whereas this is more convenient than obtaining skin biopsies for analysis, these markers are likely to be present at much lower levels in the serum than directly in affected tissue, as presumably they "leak" there from high concentrations in lesional skin and become diluted. This makes them harder to detect and likely results in more variability across patients, making it difficult to set a strict cutoff concentration that is both sensitive and specific. Importantly, as stated by the authors, ${ }^{1}$ sCD25 and sCD27 levels in the serum are not specific to vitiligo but are elevated in patients with other inflammatory conditions, including acute and chronic systemic viral infections. Thus, use of serum biomarkers may be limited to patients with no other inflammatory conditions, which may be difficult to determine. Despite these caveats, Speeckaert and colleagues ${ }^{1}$ provide some important data in a large number of patients that may translate to useful markers for future clinical trials. Additional studies of markers in the lesional skin, or potentially combining multiple serum markers, may enhance the sensitivity and specificity of these assays and provide an improved strategy to monitor disease activity and treatment responses in vitiligo.

\section{ARTICLE INFORMATION}

Author Affiliations: Division of Dermatology,

Department of Medicine, University of Massachusetts Medical School, Worcester

Corresponding Author: John E. Harris, MD, PhD Division of Dermatology, Department of Medicine University of Massachusetts Medical School, 281 Lincoln St, Hahemann Campus, Worcester, MA 01605 (john.harris@umassmed.edu).

Published Online: August 24, 2016. doi:10.1001/jamadermatol.2016.2501.

Conflict of Interest Disclosures: None reported.

\section{REFERENCES}

1. Speeckaert R, Lambert J, van Geel N. Clinical significance of serum soluble CD molecules to assess disease activity in vitiligo [published online August 24, 2016]. JAMA Dermatol. doi:10.1001 /jamadermatol.2016.2366.

2. Malek TR, Castro I. Interleukin-2 receptor signaling: at the interface between tolerance and immunity. Immunity. 2010;33(2):153-165.

3. Russell SE, Moore AC, Fallon PG, Walsh PT. Soluble IL-2Ra (sCD25) exacerbates autoimmunity and enhances the development of Th17 responses in mice. PLoS One. 2012;7(10):e47748.

4. Rubin LA, Nelson DL. The soluble interleukin-2 receptor: biology, function, and clinical application. Ann Intern Med. 1990;113(8):619-627.

5. Lens SM, Tesselaar $K$, van Oers $M H$, van Lier RA. Control of lymphocyte function through CD27-CD70 interactions. Semin Immunol. 1998;10 (6):491-499.

6. Rashighi M, Agarwal P, Richmond JM, et al CXCL10 is critical for the progression and maintenance of depigmentation in a mouse model of vitiligo. Sci Transl Med. 2014;6(223):223ra23.

7. Ciccarelli BT, Yang G, Hatjiharissi E, et al. Soluble CD27 is a faithful marker of disease burden and is unaffected by the rituximab-induced IgM flare, as well as by plasmapheresis, in patients with Waldenström's macroglobulinemia. Clin Lymphoma Myeloma. 2009;9(1):56-58

8. De Milito A, Aleman S, Marenzi R, et al. Plasma levels of soluble CD27: a simple marker to monitor immune activation during potent antiretroviral therapy in HIV-1-infected subjects. Clin Exp Immunol. 2002;127(3):486-494.

9. Ezzedine K, Eleftheriadou V, Whitton M van Geel N. Vitiligo. Lancet. 2015;386(9988):74-84
10. Rashighi $M$, Harris JE. Interfering with the IFN-y/CXCL10 pathway to develop new targeted treatments for vitiligo. Ann Transl Med. 2015;3(21): 343.

11. Ehlers MR, Nepom GT. Immune-directed therapy for type 1 diabetes at the clinical level: the Immune Tolerance Network (ITN) experience. Rev Diabet Stud. 2012;9(4):359-371.

12. Guidance for Industry: adaptive design clinical trials for drugs and biologics. Silver Spring, MD: Center for Drug Evaluation and Research, Food and Drug Administration; 2010. http://www.fda.gov /downloads/Drugs/.../Guidances/ucm201790.pdf. Accessed July 4, 2016.

13. Jones D. Adaptive trials receive boost. Nat Rev Drug Discov. 2010;9(5):345-348.

14. Wang $X X$, Wang $Q Q$, Wu JQ, et al. Increased expression of CXCR3 and its ligands in patients with vitiligo and CXCL10 as a potential clinical marker for vitiligo. Br J Dermatol. 2016;174(6):1318-1326.

15. Shi YL, Li K, Hamzavi I, Lim HW, Zhou L, Mi QS Elevated circulating soluble interleukin-2 receptor in patients with non-segmental vitiligo in North American. J Dermatol Sci. 2013;71(3):212-214. 